\title{
Assessment of the removal of side nanoparticulated populations generated during one-pot synthesis by asymmetric flow field-flow fractionation coupled to elemental mass spectrometry
}

\author{
Diego Bouzas-Ramos, Marta García-Cortes, Alfredo Sanz-Medel, Jorge Ruiz Encinar*, \\ José M. Costa-Fernández* \\ Department of Physical and Analytical Chemistry, University of Oviedo, Avda. Julián Clavería 8, 33006, Oviedo, Spain
}

\section{A R T I C L E I N F O}

\section{Article history:}

Received 7 February 2017

Received in revised form 30 June 2017

Accepted 24 August 2017

Available online 30 August 2017

\section{Keywords:}

Asymmetric flow field-flow fractionation

Quantum dots

Side nanoparticulated populations

Surface modification

Dihydrolipoic acid bidentate ligand

Inductively coupled plasma-mass

spectrometry

\begin{abstract}
A B S T R A C T
Coupling of asymmetric flow field-flow fractionation (AF4) to an on-line elemental detection (inductively coupled plasma-mass spectrometry, ICP-MS) has been recently proposed as a powerful diagnostic tool for characterization of the bioconjugation of CdSe/ZnS core-shell Quantum Dots (QDs) to antibodies. Such approach has been used herein to demonstrate that cap exchange of the native hydrophobic shell of core/shell QDs with the bidentate dihydrolipoic acid ligands directly removes completely the eventual side nanoparticulated populations generated during simple one-pot synthesis, which can ruin the subsequent final bioapplication. The critical assessment of the chemical and physical purity of the surface-modified QDs achieved allows to explain the transmission electron microscopy findings obtained for the different nanoparticle surface modification assayed.
\end{abstract}

(C) 2017 Elsevier B.V. All rights reserved.

\section{Introduction}

Quantum dots (QDs) are a group of luminescent semiconductor nanoparticles (NPs) exhibiting exceptional optical and electronic properties. QDs have attracted significant attention in many scientific research fields over the last years due to such exceptional properties as bionanoanalytical probes for biological sensing, cellular labelling and in-vitro and in-vivo imaging [1]. In spite of considerable recent progress, there is still an urgent need to yield biocompatible and high-quality QDs with superior control over their size, monodispersity stability and surface properties, and, above all, it is imperative to assess the purity of the final product [2]. In this vein, aqueous solubilization and surface functionaliza-

Abbreviations: ODs, quantum dots; NPs, nanoparticles; PQDs, polymer-coated quantum dots; DHLA, dihydrolipoic acid; DHLA-QDs, DHLA-capped quantum dots; AF4, asymmetric flow field-flow fractionation; ICP-MS, inductively coupled plasmamass spectrometry; TEM, transmission electron microscopy; DLS, dynamic light scattering; TOP, trioctylphosphine; TOPO, trioctylphosphine oxide; FIA, flow injection analysis; PdI, polydispersity index.

* Corresponding authors.

E-mail addresses: ruizjorge@uniovi.es (J.R. Encinar), jcostafe@uniovi.es (J.M. Costa-Fernández). tion remain as the limiting steps for quality assurance of the QDs employed in biological applications [3].

The most popular synthesis procedures of high quality QDs are carried out at high temperatures in organic solvents with the drawback of generating QDs coated with hydrophobic ligands (e.g. TOP/TOPO) [4]. Such QDs are not compatible with aqueous solvents, and hence, an effective modification of the QD surface with an appropriate coating is mandatory to transfer and stabilize such non-polar QDs into aqueous media. Of course, such process should assure their original colloidal, photophysical and size properties, and provide them with adequate reactive groups for further bioconjugations [5]. According to literature [6], among the general phase-transfer approaches aimed to achieving this goal, encapsulation of the nanoparticles by an amphiphilic polymer and surface ligand exchange are the most widely studied approaches. In general terms, covering the hydrophobic QDs with amphiphilic polymers results in well-dispersed QDs with a better water-stability for long periods of time than those modified using the ligand exchange method [5]. However, it has been reported that certain losses from the surface of the QDs due to the degradation of the polymer coating may occur [7], generating free polymer micelles which could worsen the quality of the polymer-coated QDs (PQDs) [8]. Alternatively, QDs surface modification via a ligand exchange of the 
native hydrophobic shell with bidentate-thiol ligands, such as dihydrolipoic acid (DHLA) [9], could significantly improve the purity grade of the aqueous-dispersed QDs avoiding the undesired desorption of polymer moieties mentioned above (so preserving the synthesized QD functionality and stability).

Considering the foregoing discourse, analytical characterization of the water-solubilized QDs is crucial to assure their quality before final functionalization with specific biomolecule recognition units (e.g. antibodies, DNA or aptamers) [3]. However, little is known so far about the level of quality and purity of the QDs after treatments to enable the sought transfer into aqueous media. In other words, a deeper understanding, control and assessment of the final QDs status after their water-solubilization is today mandatory and a carefully selected combination of complementary analytical techniques will be necessary for that purpose.

In this context, separation methods might play an important role for a more complete characterization of nanoparticles in complex mixtures. The chromatographic resolution in the nanoscale size of techniques such as size exclusion chromatography turned out to be very limited [7]. We have recently demonstrated the potential of the coupling of a soft and size-dependent separation technique (asymmetric flow field-flow fractionation, AF4) with elemental mass spectrometry detection (inductively coupled plasma-mass spectrometry, ICP-MS) to control the final quality of the products obtained in different polymer-coated QDs syntheses and to quantify QDs:antibodies bioconjugation efficiency [10]. Here, this methodology provided strong evidences explaining the different nanoparticle size distributions observed by TEM for suspensions obtained after different QDs surface-modifications.

\section{Experimental}

\subsection{Reagents and materials}

All the chemical reagents used in the experiments were of analytical grade and used as received without further purification. Deionized ultrapure water $(18.2 \mathrm{M} \Omega \mathrm{cm})$ was obtained with a MilliQ system (Millipore, Bedford, MA, USA) and used throughout the work.

The precursors used for the synthesis of the quantum dots (QDs) were selenium powder (99.99\%), cadmium oxide (99.99\%), hexamethyldisilathiane, $1.0 \mathrm{M}$ diethylzinc solution in hexanes, trioctylphosphine (TOP, 90\%), trioctylphosphine oxide (TOPO, 99\%) and anhydrous chloroform ( $\geq 99 \%$ ), all of them purchased from Sigma-Aldrich (Milwaukee, WI, USA), and hexylphosphonic acid (HPA) obtained from Alfa Aesar (Karlsruhe, Germany).

Water-solubilization of the QDs was achieved by both coating their surface with an amphiphilic polymer and, on the other hand, capping them with dihydrolipoic acid (DHLA) ligands. For such purposes, poly(isobutylene-alt-maleic anhydride), bis(6aminohexyl)amine and sodium borohydride ( $\geq 96 \%$ ) (all of them purchased from Fluka, Basel, Switzerland), and $D L$-thioctic acid (98\%) from Acros Organics (Geel, Belgium) were used. Dodecylamine, potassium tert-butoxide ( $\geq 98 \%$ ), sodium bicarbonate and anhydrous tetrahydrofuran, methanol and toluene, all acquired from Sigma-Aldrich, were also used during the solubilization processes.

Ammonium acetate and ammonia, both of them from SigmaAldrich, were used to prepare the solution employed as carrier in the asymmetric flow field-flow fractionation (AF4) experiments. Nitric acid (65\%, v/v) from Merck (Darmstadt, Germany) was additionally purified by sub-boiling distillation to operate with the minimum background levels of metallic impurities. External flow injection analysis (FIA) calibrations using Merck certified
$1000 \mathrm{mg} \mathrm{L}^{-1}$ standards of Cd, Se, S and $\mathrm{Zn}$ were used in the inductively coupled plasma-mass spectrometry (ICP-MS) analysis.

\subsection{Instrumentation}

UV-vis absorption spectra were recorded at room temperature on a Genesys 10S Spectrophotometer (Thermo Scientific, Germany). Fluorescence spectra were measured using a Varian Cary Eclipse fluorescence spectrophotometer (Agilent, Germany) ensuring a fixed excitation wavelength of $350 \mathrm{~nm}$, with both excitation and emission slit width of 5 and $10 \mathrm{~nm}$, respectively. All the measurements of absorbance and fluorescence were carried out using conventional Quartz SUPRASIL cuvettes (Hellma Analytics, Germany). Zeta potential and dynamic light scattering (DLS) measurements were carried out with a Zetasizer Nano ZS (Malvern Instruments, UK). All these measurements were performed using DTS1070 disposable capillary cells and ZEN0040 disposable cuvettes, respectively.

Transmission electron microscopy (TEM) images were obtained on a JEM-2100F (JEOL, Japan) transmission electron microscope with a copper grid, using an accelerating voltage of $200 \mathrm{kV}$. The core sizes of the QDs were measured by counting TEM images by digital analysis employing the Image software of image processing.

The AF4 system used was an AF2000 (Postnova Analytics, Germany). Regenerated cellulose membranes with $10 \mathrm{kDa}$ cutoff were used as accumulation walls. Details of the AF4 technique principles can be found elsewhere [11,12]. Table S1 (see in the Supplementary material) summarizes the channel characteristics and flow rate programs employed for fractionating the assessed samples (i.e. polymer-coated QDs and DHLA-capped QDs). After completion of the elution program, the cross flow rate was maintained at $0.0 \mathrm{~mL} \mathrm{~min}^{-1}$ (until obtaining the fractograms of $40 \mathrm{~min}$ ) to ensure the complete elution of all species. The carrier solution was filtered on $0.1 \mu \mathrm{m}$ cellulose membrane filter before its use in the AF4 instrument.

After fractionation, the AF4 effluent was coupled on-line to fluorescence, UV-vis and ICP-MS detectors. The fluorescence and UV-vis detectors coupled were the 1260 FLD and the 1260 MWD VL (Agilent, Japan), respectively. On the other hand, the elemental detector coupled on-line to the AF4 system was an 8800 Triple Quadrupole ICP-MS (Agilent, Japan). The simultaneous elemental-specific nature of the triple quadrupole ICP-MS detector allowed the interference-free detection of the co-occurring elements present in the studied QDs. Oxygen was introduced in the collision/reaction cell at a flow of $0.35 \mathrm{~mL} \mathrm{~min}^{-1}$ and so Se and $\mathrm{S}$ were detected in mass shift MS/MS mode $\left({ }^{80} \mathrm{Se}^{+} \rightarrow{ }^{96} \mathrm{SeO}^{+}\right.$and ${ }^{32} \mathrm{~S}^{+} \rightarrow{ }^{48} \mathrm{SO}^{+}$, respectively), after their reaction with oxygen in the cell. $\mathrm{Cd}$ and $\mathrm{Zn}$, which do not react with oxygen, were measured in on mass mode $\left({ }^{116} \mathrm{Cd}^{+} \rightarrow{ }^{116} \mathrm{Cd}^{+}\right.$and ${ }^{64} \mathrm{Zn}^{+} \rightarrow{ }^{64} \mathrm{Zn}^{+}$, respectively). The integration time for the targeted isotopes ${ }^{116} \mathrm{Cd},{ }^{48} \mathrm{SO},{ }^{96} \mathrm{SeO}$ and ${ }^{64} \mathrm{Zn}$ was $0.1 \mathrm{~s}$. The operation conditions were daily optimized using a tuning solution. Integration of the fractographic peaks was performed using the MassHunter software (Agilent). ICP-MS operating conditions are summarized in Table S2 (see in the Supplementary material). Computation of the different elemental molar ratios were carried out by resorting to external calibration, performed by FIA, of the different elements present in the assayed QDs. Peak area ratios obtained could be translated into elemental molar ratios using the corresponding elemental calibration curves.

\section{Results and discussion}

First of all, core/multishell CdSe/CdS-ZnS QDs have been synthesized in our lab (see in the Supplementary material), and then, transferred to aqueous media using two different water- 
solubilization approaches (see in the Supplementary material) in parallel. One aliquot of the synthesized non-polar QDs was transferred into aqueous media by using an amphiphilic polymer (based on poly(maleic anhydride) functional groups) [13]. In this method, hydrophobic alkyl chains interact with the alkyl chains of the QD ligands, and the hydrophilic groups, disposed to the external part of the nanoparticles, confer the required water stability. The second aliquot of the synthesized non-polar QDs were modified by cap exchange of the native hydrophobic shell with hydrophilic dihydrolipoic acid (DHLA) ligands [9].

\subsection{Morphological and photochemical nanoparticle behavior after surface modification}

The quality of both surface-modified QDs was then assessed and compared using standard nanoparticle characterization techniques such as UV-vis, zeta potential measurements and dynamic light scattering (DLS). UV-vis spectrophotometry (see Fig. S1 in the Supplementary material) was used to estimate the extinction coefficient and the concentration of the QDs, with good accuracy, following Pengís equation [14]. Zeta potential was measured to assess the surface charge of QDs in solution and the values obtained for the DHLA-capped QDs (DHLA-QDs) and polymer-coated QDs were $-45 \pm 2(1 \mathrm{SD}, n=5)$ and $-35 \pm 2(1 \mathrm{SD}, n=5)$, respectively. Such values indicate that both types of QDs exhibited high colloidal stability in aqueous solution $[15,16]$. Furthermore, variability in the particle size of the QDs in solution (evaluated through DLS measurements) showed that the DHLA-QDs provided a single distribution with an adequate polydispersity index (PdI) of $0.28 \pm 0.02$ (1SD,

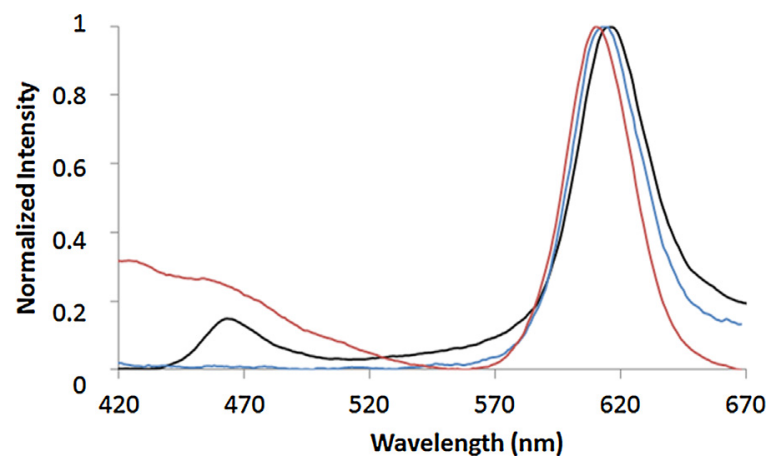

Fig. 1. Fluorescence emisFsion spectra $\left(\lambda_{\mathrm{ex}}=350 \mathrm{~nm}\right)$ of the synthesized non-polar CdSe/CdS-ZnS QDs (line in black) in chloroform, and the polymer-coated QDs (line in red) and DHLA-capped QDs (line in blue) in water media. (For interpretation of the references to colour in this figure legend, the reader is referred to the web version of this article.)

$n=5)$. On the other hand, the PdI for the PQDs was $0.35 \pm 0.01$ (1SD, $n=5$ ), a value indicating a significantly higher polydispersity than that obtained for the DHLA-QDs (all morphological parameters are summarized in Table S3 in the Supplementary material).

Additionally, both types of coated QDs under evaluation were characterized also by fluorescence spectroscopy. Fluorescence spectra (Fig. 1) of the synthesized non-polar QDs and of the both types of water-solubilized QDs were recorded under the same instrumental conditions $\left(\lambda_{\mathrm{ex}}=350 \mathrm{~nm}\right)$.

It can be observed that the three assessed QD samples displayed an intense sharp emission band at $610 \mathrm{~nm}$ corresponding to the
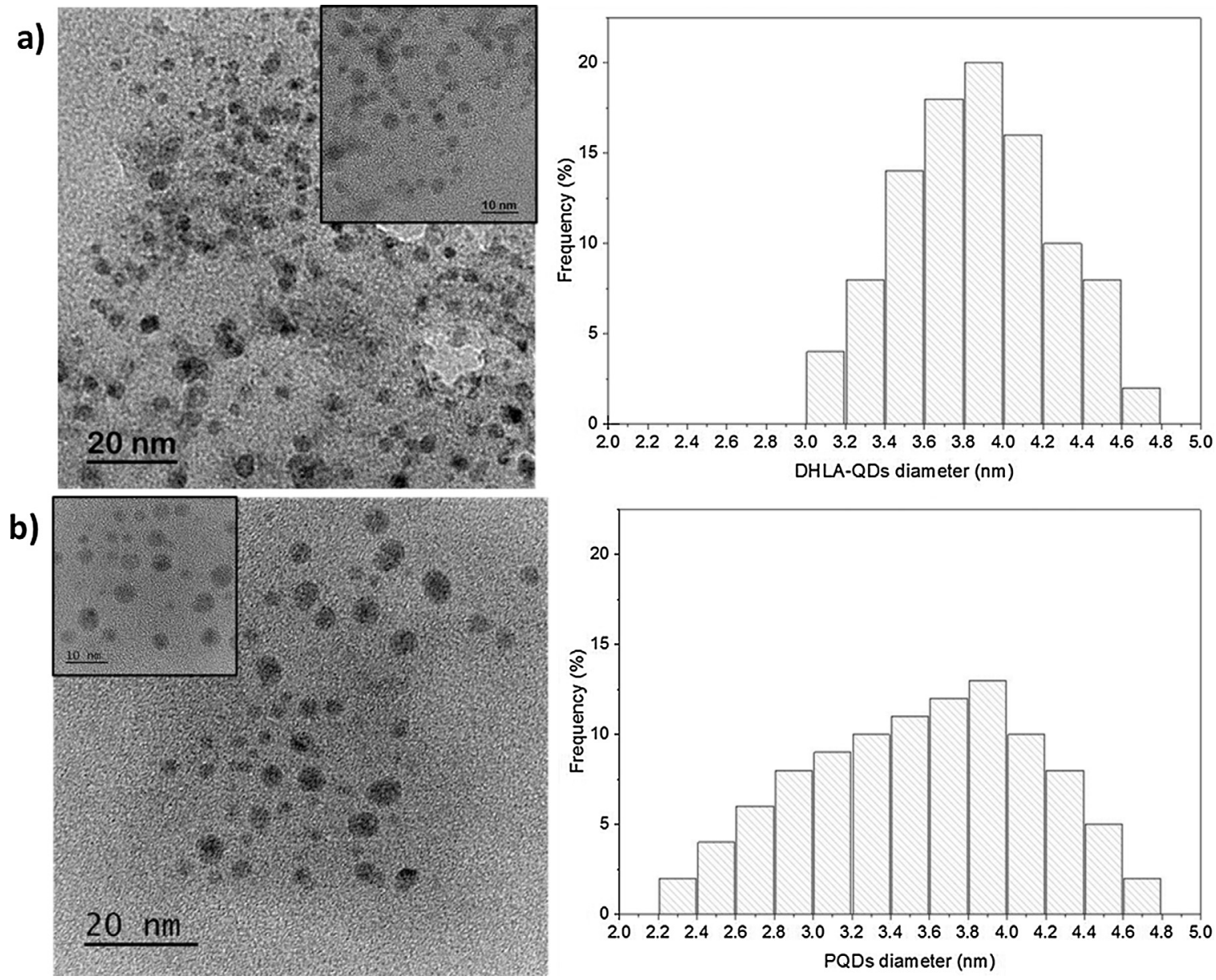

Fig. 2. TEM images and nanoparticle size distribution histograms of the (a) DHLA-capped QDs, and (b) polymer-coated QDs. 
fluorescence of the CdSe/CdS-ZnS QDs. Additionally, full width at half maximum (FWHM) of the emission peak is between 30 and $35 \mathrm{~nm}$ for the three assessed QD samples, indicating low size dispersion $[8,13]$ comparable with typical commercial QDs. However, an undesired unexpected fluorescence emission, with a maximum at $460 \mathrm{~nm}$, is observed in the synthesized non-polar QDs spectrum. This less intense emission band could be attributed to the presence of impurities or NPs of different size or nature formed mostly during the synthesis of the core/multishell QDs. Moreover, an undesirable fluorescence emission in the range between 420 and $540 \mathrm{~nm}$ is also apparent in the PQDs spectrum. As it was already shown in a previous work carried out in our lab [8], this fluorescence emission background could be originated from the photoluminescence emission of residual free polymer chains (fluorescence spectrum of the free polymer is shown in Fig. S2 in the Supplementary material) or coexisting less abundant NP populations emitting at lower wavelengths. Of course, a combination of the two possibilities is also very likely to occur. Again, there is no evidence of the presence of such secondary emission band in the fluorescence spectrum of the DHLA-QDs and only the expected fluorescence band centered at $610 \mathrm{~nm}$ is observed.

To clarify this issue, the size distribution of the QDs core after both phase-transfer approaches was studied using TEM. As can be seen in Fig. 2a, the capping with DHLA led to a well-defined NP population with diameters ranging from 3.0 to $4.8 \mathrm{~nm}$ (average size of $3.82 \pm 0.37 \mathrm{~nm}$; $1 \mathrm{SD}, n=50$ ). Conversely, water-solubilization with amphiphilic polymer (Fig. 2b) provided a more disperse nanoparticle population with a lower average size $(3.51 \pm 0.59 \mathrm{~nm}$; $1 \mathrm{SD}$, $n=50$ ). Of course, the greatest polydispersity of the PQDs could be likely related to the undesired secondary less intense band observed in its fluorescence spectrum.

\subsection{Influence of nanoparticle surface coating on chemical purity}

We resorted to the on-line coupling of the AF4 separation technique with both molecular and elemental detectors to assess the chemical nature of both surface-modified QDs samples, thus allowing to explain the TEM findings obtained (see Fig. 2a and b).

The UV-vis and fluorescence detectors were used to monitor any absorption and fluorescence emission, respectively, from the QDs as well as any unexpected species present in the solubilized-QD suspensions. On the other hand, an ICP-MS equipped with a triple quadrupole was selected as elemental detector to enable sensitive [17] and reliable simultaneous quantification of the elemental constituents of the CdSe/CdS-ZnS QDs (including elements difficult to be detected by ICP-MS such as sulphur), as well as other possible NP populations eventually obtained.

In agreement with the results recently reported by MenéndezMiranda et al. [8], the fractograms obtained for PQDs with fluorescence and UV-vis detection (Fig. 3a) indicate the presence of at least two different species. Conversely, when DHLA were used as ligands for QDs capping only one fluorescence and one UV-vis
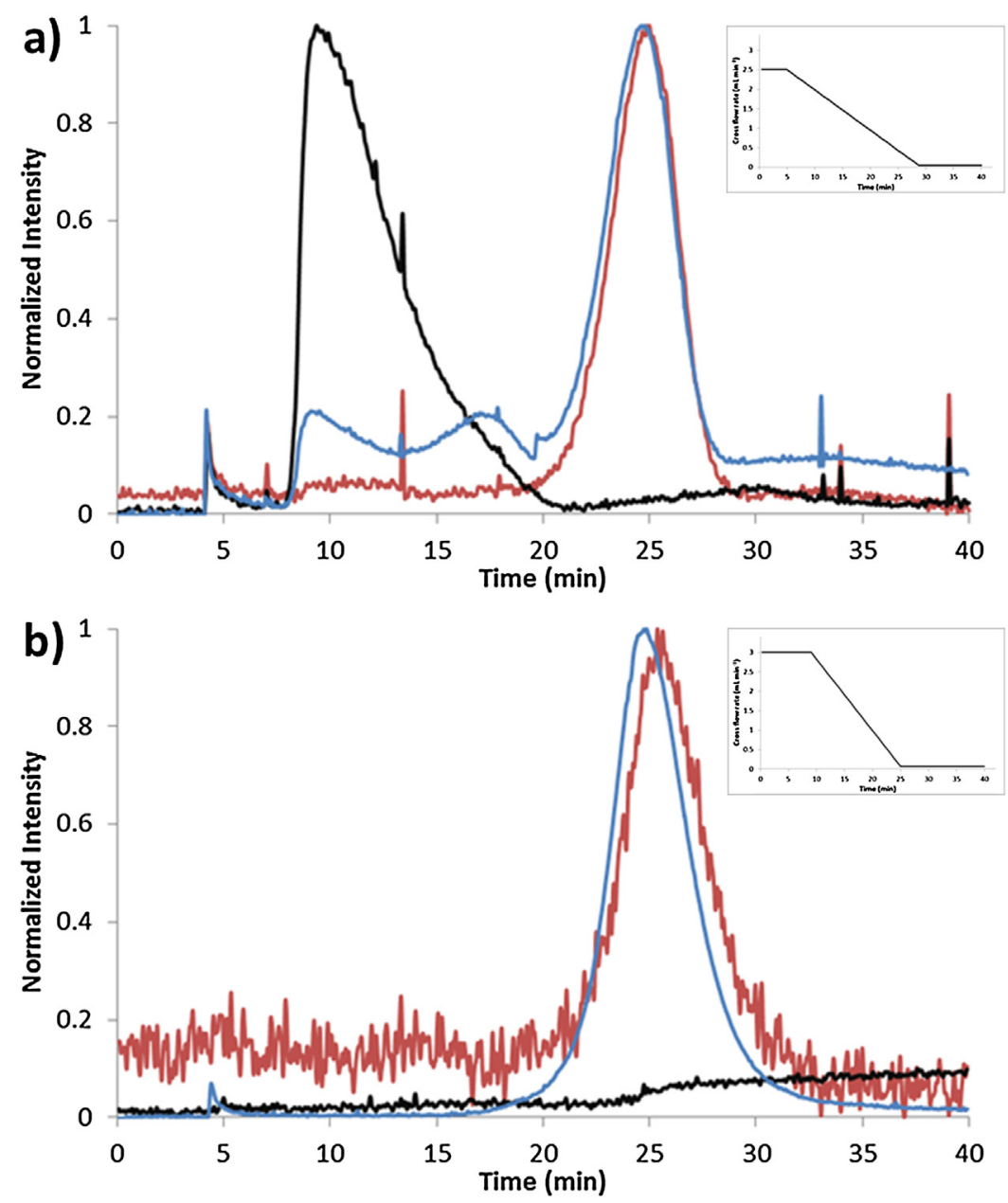

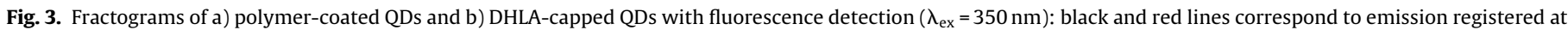

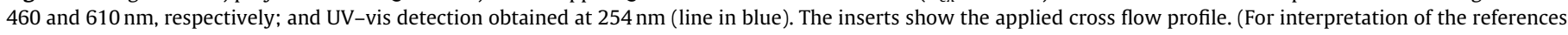
to colour in this figure legend, the reader is referred to the web version of this article.) 
bands (Fig. 3b) appeared at the retention time of $25 \mathrm{~min}$, indicating the presence of a single population of NPs. In addition, no photoluminescence emission at $460 \mathrm{~nm}$ was detected in this case. These results are in agreement with the greater polydispersity observed in TEM for the PQDs in comparison with the DHLA-capped QDs. However, the results obtained up to this point do not provide information about the identity of the different populations detected. In this sense, the triple quadrupole ICP-MS detection (see Fig. 4 for results) allowed us to get more information on the identity and the chemical nature of these different NP populations. In this context, the obtained ICP-MS fractogram of the PQDs (Fig. 4a) showed the expected CdSe/CdS-ZnS QDs eluting at $25 \mathrm{~min}$ and containing significant amounts of the four elements (Cd, Se, S, Zn) monitored.

As shown by Fig. 4a, at lower hydrodynamic size ( $\sim 18 \mathrm{~min})$ a second NP population eluted. It consisted of independent CdS NPs, which are known to have smaller size than the core/multishell QDs [18]. The Cd/S molar ratio computed in this peak, $1.27 \pm 0.09$ (1SD, $n=3$ ), showed a slight excess of the metallic element, a common feature that has already been observed in other unshelled nanoparticles $[19,20]$. Elemental ICP-MS fractogram also showed that a broad and undefined band eluted between 10 and $20 \mathrm{~min}$, which had a strong fluorescent emission at $460 \mathrm{~nm}$ (Fig. 3a). The elemental composition of such species revealed that they contained Cd and, mostly, $\mathrm{Zn}$. It is well-known that, depending on the environmental conditions used, certain losses of the used polymer coating from the surface of the QDs take place [7,8] and such polymer residues or micelles emit strong luminescence at around $460 \mathrm{~nm}$ (see Fig. S2 in the Supplementary material). In this context, the presence of a significant $\mathrm{Zn}$ signal at this eluting time can be explained considering the strong affinity for free metals of the maleic acid-based polymers [21] used in this solubilization procedure, $\mathrm{Zn}(\mathrm{II})$ and $\mathrm{Cd}(\mathrm{II})$ in par- ticular, likely to be present as core/shell reagent excesses or after releasing from the multishell of the QDs, that could then react with the maleic-containing polymers.

In contrast, the absence of any undesirable species when DHLA ligands were used to water-solubilize QDs was also confirmed by the ICP-MS fractogram (Fig. 4b). In this case, only the peak of the CdSe/CdS-ZnS QDs appeared at the retention time of $25 \mathrm{~min}$ (see Fig. 4b). In this case, not only the polymer micelles signals disappeared but also the extra CdS NP population observed for the polymer-coated QDs. This fact seems to point a possible purification effect which takes place when engineered QDs are water-solubilized via ligand exchange using DHLA.

It is very interesting now revisiting the TEM images shown in Fig. 2 under the light of the chemical information provided by Fig. 3 and particularly Fig. 4. It can be seen that the use of the hybrid technique enabled to unveil the NP population observed with lower diameters ( 2.2-3.5 nm) observed in the PQDs (Fig. 2b): a different type of NPs composed of Cd and S (Fig. 4a).

Conversely, it seems that the use of DHLA ligands for capping QDs removed the presence of such CdS NPs impurities, leading to a purified population of high-quality DHLA-capped QDs as has been demonstrated by both TEM (Fig. 2a) and AF4-ICP-MS (Fig. 4b). In this regard, it seems that the unexpected CdS NPs could have been formed in parallel during the shell formation over the QD core and such residual NPs were also covered in-situ by the TOP/TOPO ligands present. These TOP/TOPO ligands can be intercalated with the hydrophobic side chains of the amphiphilic polymer, resulting in the solubilization of the QDs and, also, of the undesired CdS NPs population observed in Fig. 4a. Conversely, the ligand exchange process using DHLA seems to occur effectively only with the CdSe/CdS-ZnS QDs while it does not take place at all with the
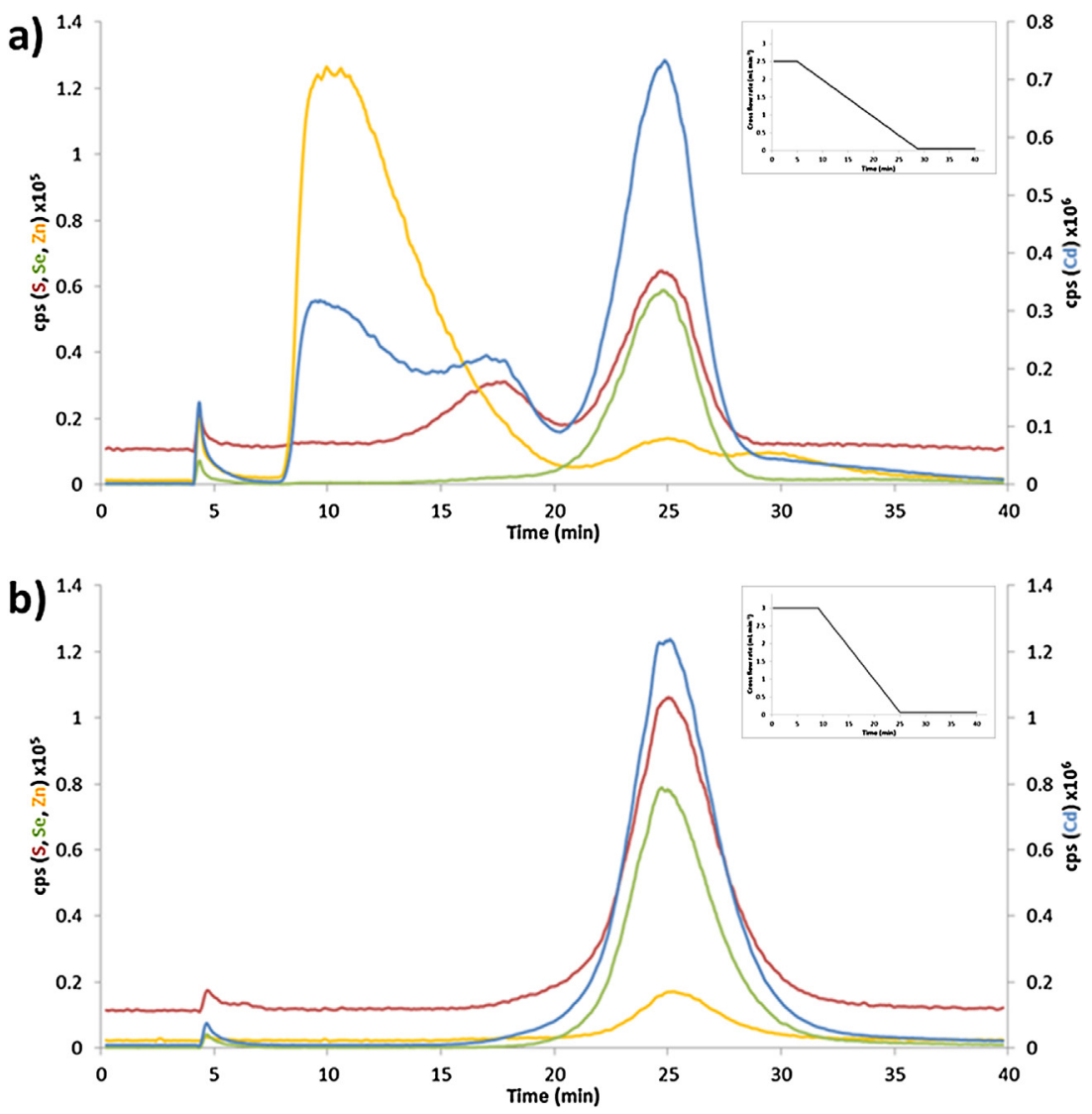

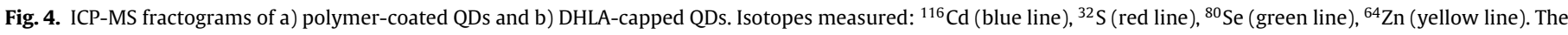
inserts show the applied cross flow profile. (For interpretation of the references to colour in this figure legend, the reader is referred to the web version of this article.) 
CdS NPs present as impurities (see Fig. 4b). This finding may be likely due to the fact that the thiol groups of the DHLA ligands exhibit a much greater affinity to Zn-containing shells (e.g. the CdSZnS mixed-shell of the QDs synthesized) than to bare core NPs [22], such as the CdS NPs. These undesirable CdS NPs could not be able to withstand the ligand exchange process and hence not being watersolubilized, leading to the entire loss of stability of these residual NPs, and thereby yielding a purified suspension of water-stabilized DHLA-capped QDs of higher quality.

Interestingly, we can also use the AF4 coupled to ICP-MS detector to demonstrate the presence of the DHLA ligands onto the surface of the QDs. For that purpose, external calibration, performed by flow injection analysis (FIA), of the different elements present in the QDs could be used to translate the area ratios obtained in both QD peaks (peaks at $\sim 25$ min in Fig. $4 \mathrm{a}$ and b) into elemental molar ratios. In this sense, as expected, the computed Cd/Se molar ratio remained virtually constant (results are not statistically different) when using either the polymer $(2.76 \pm 0.06$; $1 \mathrm{SD}, n=3)$ or the DHLA ligands $(2.81 \pm 0.08 ; 1 \mathrm{SD}, n=3)$. This could be ascribed to the fact that $\mathrm{Cd}$ and Se are present in the core and the shell of the QDs, and were not affected by the ligand exchange procedure on the nanoparticle surface. In contrast, the added DHLA molecules produced ligand exchange and so brought an increase of the sulphur atoms on the surface of the QDs. Therefore, a significant difference in the computed $\mathrm{Cd} / \mathrm{S}$ molar ratios was observed comparing PQDs and DHLA-QDs. Interestingly, the calculated $\mathrm{Cd} / \mathrm{S}$ molar ratio decreased significantly from $2.28 \pm 0.06$ to $1.59 \pm 0.09$ (1SD, $n=3$ ), respectively. This finding confirms that the DHLA ligands were attached to the surface of the DHLA-capped QDs. Moreover, the herein presented approach allowed also estimating the nanoparticle ligand density, which is of great importance and technically challenging [20] (see the Supplementary material).

\section{Conclusions}

The final quality of QDs obtained using the two more common water-solubilization approaches (amphiphilic polymer and ligand exchange with DHLA) has been assessed using a timeresolved nano-sized separation technique, AF4, coupled on-line with both elemental and molecular detection. The AF4-ICP-MS coupling enabled to get invaluable information on the purity and stability as well as the chemical composition of the derivatized QDs obtained.

Interestingly, it was observed that a purification effect, aside from the sought water-solubilization, occurs when the QDs were capped with DHLA ligands via ligand exchange. This approach led to QDs with higher chemical purity in comparison with those coated with the amphiphilic polymer, an approach providing undesirable nanoparticulated species coexisting with the desired QDs. In fact, multiple-steps synthesis, post-production processing and further purification steps are typically required to remove most of such undesired impurities. These results could be of great practical value in order to simplify the production of high quality engineered QDs, which could be used for example in further analytical and bioanalytical photoluminescent QD-based applications.

\section{Acknowledgements}

This work was supported by Spanish Ministry of Education (CTQ2016-79412-P), Principado de Asturias (FC-15-GRUPIN14092) and Agilent Technologies Foundation is also acknowledged. Diego Bouzas-Ramos and Marta García-Cortes acknowledge the Ph.D. grants (BP14-137 and BP13-110, respectively) from Principado de Asturias (Spain).

\section{Appendix A. Supplementary data}

Supplementary data associated with this article can be found, in the online version, at http://dx.doi.org/10.1016/j.chroma.2017.08. 068.

\section{References}

[1] K.D. Wegner, N. Hildebrandt, Quantum dots: bright and versatile in vitro and in vivo fluorescence imaging biosensors, Chem. Soc. Rev. 44 (2015) 4792-4834.

[2] S.J. Soenen, P. Rivera-Gil, J.M. Montenegro, W.J. Parak, S.C. De Smedt, K. Braeckmans, Cellular toxicity of inorganic nanoparticles: common aspects and guidelines for improved nanotoxicity evaluation, Nano Today 6 (2011) 446-465.

[3] A.S. Karakoti, R. Shukla, R. Shanker, S. Singh, Surface functionalization of quantum dots for biological applications, Adv. Colloid Interface Sci. 215 (2015) 28-45.

[4] Z.A. Peng, X. Peng, Formation of high-quality CdTe CdSe, and CdS nanocrystals using CdO as precursor, J. Am. Chem. Soc. 123 (2001) 183-184.

[5] A.M. Smith, H. Duan, A.M. Mohs, S. Nie, Bioconjugated quantum dots for in vivo molecular and cellular imaging, Adv. Drug Deliv. Rev. 60 (2008) 1226-1240.

[6] H. Mattoussi, G. Palui, H. Bin Na, Luminescent quantum dots as platforms for probing in vitro and in vivo biological processes, Adv. Drug Deliv. Rev. 64 (2012) 138-166

[7] L. Trapiella, A.R.M. Bustos, J.R. Encinar, J.M. Costa, R. Pereiro, A. Sanz-Medel, New integrated elemental and molecular strategies as a diagnostic tool for the quality of water soluble quantum dots and their bioconjugates, Nanoscale 3 (2011) 954-957

[8] M. Menéndez-Miranda, M.T. Fernández-Argüelles, J.M. Costa-Fernández, J.R. Encinar, A. Sanz-Medel, Elemental ratios for characterization of quantum-dots populations in complex mixtures by asymmetrical flow field-flow fractionation online coupled to fluorescence and inductively coupled plasma mass spectrometry, Anal. Chim. Acta 839 (2014) 8-13.

[9] A.R. Clapp, E.R. Goldman, H. Mattoussi, Capping of CdSe-ZnS quantum dots with DHLA and subsequent conjugation with proteins, Nat. Protoc. 1 (2006) 1258-1266.

[10] M. Menéndez-Miranda, J. Ruiz Encinar, J.M. Costa-Fernández, A. Sanz-Medel, Asymmetric flow field-flow fractionation coupled to inductively coupled plasma mass spectrometry for the quantification of quantum dots bioconjugation efficiency, J. Chromatogr. A 1422 (2015) 247-252.

[11] G. Yohannes, M. Jussila, K. Hartonen, M.L. Riekkola, Asymmetrical flow field-flow fractionation technique for separation and characterization of biopolymers and bioparticles, J. Chromatogr. A 1218 (2011) 4104-4116.

[12] K.G. Wahlund, Flow field-flow fractionation: critical overview, J. Chromatogr. A 1287 (2013) 97-112

[13] M.T. Fernández-Argüelles, A. Yakovlev, R.A. Sperling, C. Luccardini, S. Gaillard, A. Sanz-Medel, J.M. Mallet, J.C. Brochon, A. Feltz, N. Oheim, W.J. Parak, Synthesis and characterization of polymer-coated quantum dots with integrated acceptor dyes as FRET-based nanoprobes, Nano Lett. 7 (2007) 2613-2617.

[14] W.W. Yu, L. Qu, W. Guo, X. Peng, Experimental determination of the extinction coefficient of CdTe CdSe, and CdS nanocrystals, Chem. Mater. 15 (2003) 2854-2860

[15] S. Honary, F. Zahir, Effect of zeta potential on the properties of nano-drug delivery systems - a review (Part 2), Trop. J. Pharm. Res. 12 (2013) 265-273.

[16] H. Ohshima, The Derjaguin-Landau-Verwey-Overbeek (DLVO) theory of colloid stability, in: H. Ohshima (Ed.), Electrical Phenomena at Interfaces and Biointerfaces: Fundamentals and Applications in Nano-, Bio-, and Environmental Sciences, John Wiley \& Sons, Inc., 2012, pp. 27-34.

[17] S.D. Fernández, N. Sugishama, J.R. Encinar, A. Sanz-Medel, Triple quad ICPMS (ICPQQQ) as a new tool for absolute quantitative proteomics and phosphoproteomics, Anal. Chem. 84 (2012) 5851-5857.

[18] L. Qi, H. Cölfen, M. Antonietti, Synthesis and characterization of CdS nanoparticles stabilized by double-hydrophilic block copolymers, Nano Lett. 1 (2001) 61-65.

[19] A.R.M. Bustos, J.R. Encinar, M.T. Fernández-Argüelles, J.M. Costa-Fernández, A. Sanz-Medel, Elemental mass spectrometry: a powerful tool for an accurate characterisation at elemental level of quantum dots, Chem. Commun. (2009) 3107-3109.

[20] D. Bouzas-Ramos, M. Menéndez, J.M. Costa-Fernández, J.R. Encinar, A. Sanz-Medel, Precise determination of the nanoparticle concentration and ligand density of engineered water-soluble HgSe fluorescent nanoparticles, RSC Adv. 6 (2016) 19964-19972.

[21] R. Hasanzadeh, P.N. Moghadam, N. Samadi, Synthesis and application of modified poly (styrene-alt-maleic anhydride) networks as a nano chelating resin for uptake of heavy metal ions, Polym. Adv. Technol. 24 (2013) 34-41.

[22] A.F.E. Hezinger, J. Teßmar, A. Göpferich, Polymer coating of quantum dots - a powerful tool toward diagnostics and sensorics, Eur. J. Pharm. Biopharm. 68 (2008) 138-152. 